Relations industrielles

Industrial Relations

\title{
Le Canada, dernière chance de l'Europe par Claude Julien. Grasset, Paris, 1965, 255 pages.
}

\section{Gérard Dion}

Volume 21, numéro 2, 1966

URI : https://id.erudit.org/iderudit/027692ar

DOI : https://doi.org/10.7202/027692ar

Aller au sommaire du numéro

Éditeur(s)

Département des relations industrielles de l'Université Laval

ISSN

0034-379X (imprimé)

1703-8138 (numérique)

Découvrir la revue

Citer ce compte rendu

Dion, G. (1966). Compte rendu de [Le Canada, dernière chance de l'Europe par Claude Julien. Grasset, Paris, 1965, 255 pages.] Relations industrielles /

Industrial Relations, 21(2), 306-307. https://doi.org/10.7202/027692ar

Tous droits réservés @ Département des relations industrielles de l'Université Laval, 1966
Ce document est protégé par la loi sur le droit d'auteur. L'utilisation des services d'Érudit (y compris la reproduction) est assujettie à sa politique d'utilisation que vous pouvez consulter en ligne.

https://apropos.erudit.org/fr/usagers/politique-dutilisation/ 
Puis en passant à la partie IV on retrouve les contributions cette fois de la sociologie industrielle et des découvertes et expériences dont elle a été le cadre (chap. 12), l'influence de la dépression économique (chap. 13) et de la seconde guerre (chap. 14) sur les différentes fonctions concernant la direction du personnel.

Somme toute ce volume pourra être de lecture intéressante et très utile d̀ tout étudiont, professeur ou professionnel intéressé à la direction du personnel, à la théorie de l'organisation, ò I'histoire des affaires, d̀ I'histoire économique ou à l'économique du travail.

\section{Ronald Pleau}

The Price Systems and Resource Allocation (3rd Edition), Richard $H$. Leftwich, Holt, Rinehart and Winston of Canada Ltd.

833 Oxford St., Toronto 18, Ont., 1966, 369 pages.

L'auteur expose comment le mécanisme des prix alloue les ressources rares de l'économie parmi ses besoins illimités. A cet égard, ce volume ne présente que peu de nouveau, si on le compare aux nombreux outres parus depuis plusieurs années sur le même sujet.

Nous sommes très heureux d'ajouter que ce volume se distingue cependant de plusieurs de ses semblables par la qualité de son exposé, clair, concis et complet. L'auteur fait preuve d'une excellente pédagogie. Et celle-ci sera d'autant plus appréciée des étudiants de l'économique que le contenu du volume se situe à un niveau, plutôt élémentaire de la micro-économique.

Signalons aussi l'intérêt du dernier chapitre qui représente la programmation linéaire. En effet, tout le monde sait que l'équilibre de la firme peut être déterminé par le marginalisme orthodoxe, d̀ la condition, toutefois, qu'il n'existe pas de limitations dans la quantité des facteurs de production utilisés. Or, de telles limitations constituent probablement la règle générale. C'est d'ailleurs ce chapitre qui constitue l'addition majeure de cette troisième édition.

Nous recommandons donc fortement cet excellent volume d̀ tous les intéressés d̀ ce sujet primordial en économique qu'est le mécanisme des prix et l'allocation des ressources.

Bertrand Belzile
Le Canada, dernière chance de l'Europe par Claude Julien. Grasset, Paris, 1965. 255 pages.

Cet ouvrage est d'abord écrit en fonction de lecteurs européens et porticulièrement des responsables de la politique en France.

L'auteur y développe la thèse suivante. Le destin de l'Europe est lié au sort du Canada. « Une fois dans son histoire la France a perdu le Canada au profit de l'Angleterre. De nos jours, si elles n'y prennent garde, la France et l'Angleterre vont perdre le Conada au profit des Etats-Unis. Et cette perte, pour l'Europe entière, serait infiniment plus grave que ne le fut pour la France la défoite de Montcalm. (53) Le Canada possède un énorme potentiel économique qui s'il venait à s'ajouter à la puissance des Etats-Unis interdirait tout équilibre entre les deux rives de I'Atlantique. Déjà le Canada est sous l'emprise des capitaux américains, mais la partie n'est pas perdue, car il y a encore place pour de vastes apports de capitaux, de techniciens et de travailleurs spécialisés.

Les lecteurs canadiens de langue française comme ceux de langue anglaise y trouveront aussi un appel au sens de la responsabilité à l'échelle mondiale. «Obnubilés par les difficiles relations entre le groupe onglophone et le groupe francophone, ils en viennent à oublier parfois leur problème numéro un, qu'ils connoissent pourtant bien, celui de leur dépendance éconornique, et donc politique, à l'égard des Etats-Unis. * (60). "Un Canada morcelé deviendrait pour l'emprise du capital américain une proie encore plus facile. L'indépendance du $\mathrm{Ca}$ nada, qui conditionne ainsi une certaine conception de l'Occident, dépend donc à la fois de l'Europe et des Canadiens euxmêmes. II est grand temps pour l'Europe de s'intéresser utilement au Canada en développant avec lui ses échanges commerciaux, en lui apportant des capitaux et des techniciens. II est grand temps, pour les Canadiens, d'envisager leurs propres problèmes dans une perspective non pas étroitement nationale, mais mondiale. w (61). On en vient ainsi à voir le rôle que le Québec est appelé à jouer sur l'avenir du monde, car c'est lui qui conteste les structures conadiennes mal adaptées aux nécessités présentes. C'est lui aussi qui peut jouer un rôle de moteur et qui est dे l'origine de solutions neuves.

Dans l'élaboration de sa thèse, l'auteur onolyse les différents éléments de la situotion économique, sociale et politique du 
Canada. On pourra peut-être le chicaner sur des questions de détails, mais l'ensemble est sérieux et très imposant.

Nous espérons que cet appel à la conscience des responsabilités tant chez les Canadiens que chez les Européens trouvero un écho.

\section{Gérard Dion}

Monagement Operations Research, Norbert Lloyd Enrick, Holt., Rinehart $\mathcal{E}$ Winston, Inc., 833 Oxford St., Toronto 18, Ont. 1965. 320 pages.

Qu'ils le veuillent ou pas, les dirigeants de l'entreprise devront avoir de plus en plus recours à la recherche opérationnelle. En effet cette méthode scientifique s'inscrit dans la méthodologie vers une science de l'action et les dirigeants, qui sont avant tout des hommes d'action qui prennent continuellement des décisions dans leur entreprise, devront faire appel da cette méthode des modèles mathématiques qui permet précisément de mieux préparer les décisions.

II ne faut quand même pas penser que la recherche opérationnelle peut se substituer complètement aux dirigeants. Certes les ordinateurs prennent plusieurs décisions en vertu des programmes préalablement établis par les dirigeants, mais ces derniers auront toujours à prendre de nom- breuses décisions et surtout les plus difficiles.

C'est donc à juste titre que dans la première partie de son volume, l'auteur définit le rôle de la recherche opérationnelle dans l'administration moderne et traite des méthodes comptables qui $y$ sont utilisées; il soulève également le problème épineux de la prise de décision face à l'incertitude.

Depuis quelques années, la littérature compte de nombreux ouvrages sur l'utilisation de la recherche opérationnelle dans la gestion des entreprises. Le contenu de tels ouvrages varie toutefois assez fortement les uns des autres. La distinction porte à la fois sur les problèmes étudiés et sur les modèles mathématiques présentés. $M$. Enrick, pour so part, s'attarde à quelques techniques importantes: dons la deuxième partie, il expose la programmation linéaire et la méthode du chemin critique; dans la troisième, il présente le modèle des phénomènes d'attente dans son utilisation pour la gestion des inventaires; et dans la dernière partie, l'auteur discute de l'échantillonnage et de l'analyse statistique.

Les faibles prérequis en mathématiques rend cet excellent volume accessible aux étudiants en administration de l'entreprise et à la plupart de nos chefs d'entreprises. Nous le conseillons à tous les intéressés.

Bertrand Belzile

\section{PUBLICATIONS RÉCENTES}

\section{GENERALITES}

«Revolucion, reforma y conservatismo: tipos de politicas agrarias en Latinoamerica $\$$, Oscar Delgado, Revista Brasileira de Ciencias Sociais, Julho 1963, Vol. III, Num. 2, pp. 172-252.

«Le deuxième congrès mondial de la population. 》, Belgrade, 30 août au 10 septembre 1965, en collaboration, Population, 20e année, no 6, novembre-décembre 1965, pp. $941-1186$.

«L'histoire de la population et de la structure sociale », par Peter Laslett, Revue internationale des sciences sociales, $\mathrm{Vol}$. XVII, (1965), no 4, pp. 626-640.
«Mutations de la propriété 》, André Tunc, Guy Houist, Louis Estrangin, André Piette, numéro spécial de Recherche sociale, no 2, novembre-décembre 1965, pp. 1-48.

«Popperism: The Scarcity of Reason 》, by James Petras, Science $\&$ Society, Vol. XXX, No. 1, Winter, 1966, pp. 1-11.

«An Armour Automation Report», by Eaton $H$. Conant, Monthly Labor Review, Vol. 88, No. 11, November 1965, pp. 12971302.

«Considérations sur l'évolution actuelle du capitalisme 》, LuC Bourcier de Carbon, Droit Social, no 1, janvier 1966, pp. 1-15. 